\title{
Relação de chuvas e casos de criptosporidiose nas mesorregiões de Pernambuco,
}

\section{Brasil}

\author{
Ratio of rain and cases of cryptosporidiosis in the mesoregions of Pernambuco, Brazil \\ Relación de lluvia y casos de criptosporidiosis en el mesoregiones de Pernambuco, Brasil
}

Recebido: 10/09/2021 | Revisado: 20/09/2021 | Aceito: 29/09/2021 | Publicado: 01/10/2021

Inalda Maria de Oliveira Messias

ORCID: https://orcid.org/0000-0001-8699-3717

Universidade Federal Rural de Pernambuco, Brasil

E-mail: inalda.messias@upe.br

José Henrique Vanderlei Oliveira

ORCID: https://orcid.org/0000-0002-3167-2761

Universidade de Pernambuco, Brasil

E-mail: Henrique.oliv@ hotmail.com

Silvan de Fátima Ferreira da Silva

ORCID: https://orcid.org/0000-0003-4421-6228 Universidade de Pernambuco, Brasil

E-mail: silvana.ferreira@upe.br

Júlio Brando Messias

ORCID: https://orcid.org/0000-0001-6996-974X Universidade de Pernambuco, Brasil

E-mail: julio.messias@upe.br

Adriano Gomes Ferreira

ORCID: https://orcid.org/0000-0003-3696-1623

Universidade Federal de Pernambuco, Brasil E-mail: adrianogomes@ymail.com

Daniela de Araújo Viana Marques

ORCID: https://orcid.org/0000-0002-2380-7910 Universidade de Pernambuco, Brasil E-mail: daniela.viana@upe.br

Ana Lúcia Figueiredo Porto

ORCID: https://orcid.org/0000-0001-5561-5158

Universidade Federal Rural de Pernambuco, Brasil E-mail: analuporto@yahoo.com.br

\begin{abstract}
Resumo
A criptosporidiose é uma doença reemergente, responsável por graves problemas de saúde pública em todo o mundo, causada pelo protozoário Cryptosporidium spp. com alto poder zoonótico, podendo contaminar alimentos, água e solo e causar surtos de diarréia, levando a perdas econômicas e sociais. O objetivo deste estudo foi avaliar a ocorrência de criptosporidiose em pacientes atendidos em um hospital público de Recife-PE. Foram avaliados 247 registros no período de 2012 a 2020, com 89 casos de criptosporidiose. Para confirmar o diagnóstico, foram utilizadas as técnicas de concentração de Ritchie e coloração de Kinyoun. Observou-se uma relação entre a precipitação e a ocorrência de criptosporidiose, o que foi confirmado entre o registro do número de casos e a precipitação na região com o maior número de casos. A pesquisa não revelou diferenças estatísticas entre os sexos ou a idade entre os casos, mas divergiu de outros estudos quando mostrou que os grupos de pacientes eram os adultos mais velhos $(75,3 \%)$ e os idosos $(16,9 \%)$. Não podemos descartar a hipótese de que o número de Unidades de Pronto Atendimento da Gerência Regional de Saúde do possa influenciar a chance de diagnóstico da doença. Mais estudos serão necessários para desmistificar sua ocorrência em idosos, bem como sua notificação.
\end{abstract}

Palavras-chave: Chuva; Cryptosporidim; Parasitologia; Diarreia.

\begin{abstract}
Cryptosporidiosis is a reemerging disease, responsible for serious public health problems worldwide, caused by the protozoan Cryptosporidium spp. with high zoonotic power, being able to contaminate food, water, and soil, and causing outbreaks of diarrhea, leading to economic and social losses. The objective of this study was to evaluate the occurrence of cryptosporidiosis in patients seen at a public hospital in Recife-PE. 247 records were evaluated from 2012 to 2020, with 89 cases of cryptosporidiosis. To confirm the diagnosis, Ritchie concentration and Kinyoun staining techniques were used. It was observed that there is a relationship between rainfall and the occurrence of cryptosporidiosis, which was confirmed between the registration of the number of cases and rainfall in the region with
\end{abstract}


the highest number of cases. The research did not reveal statistical differences between sexes or ages between cases, but it diverged from other studies when it showed that the groups of patients were the oldest adults $(75,3 \%)$ and the elderly (16,9\%). We cannot rule out the hypothesis that the number of Emergency Units -UPAs by Regional Health Management can influence the chance of disease diagnosis. Further studies will be necessary to demystify its occurrence in the elderly, as well as its notification.

Keywords: Rai; Cryptosporidium; Parasitology; Diarrhea.

\section{Resumen}

La criptosporidiosis es una enfermedad reemergente, responsable de graves problemas de salud pública en todo el mundo, causada por el protozoo Cryptosporidium spp. con alto poder zoonótico, puede contaminar los alimentos, el agua y el suelo y provocar brotes de diarrea, lo que ocasiona pérdidas económicas y sociales. El objetivo de este estudio fue evaluar la aparición de criptosporidiosis en pacientes tratados en un hospital público de Recife-PE. Se evaluaron un total de 247 registros de 2012 a 2020, con 89 casos de criptosporidiosis. Para confirmar el diagnóstico se utilizaron técnicas de concentración de Ritchie y tinción de Kinyoun. Se observó una relación entre la precipitación y la ocurrencia de criptosporidiosis, lo que se confirmó entre el registro del número de casos y la precipitación en la región con mayor número de casos. La encuesta no reveló diferencias estadísticas entre sexos o edad entre los casos, pero difirió de otros estudios cuando mostró que los grupos de pacientes eran los adultos mayores $(75,3 \%)$ y los ancianos $(16,9 \%)$. No podemos descartar la hipótesis de que el número de Unidades de Urgencias de la Dirección Regional de Salud pueda influir en la posibilidad de diagnosticar la enfermedad. Se necesitarán más estudios para desmitificar su ocurrencia en los ancianos, así como su notificación.

Palabras clave: Lluvia; Criptosporidim; Parasitología; Diarrea.

\section{Introdução}

As parasitoses intestinais representam um dos maiores problemas de saúde pública do Brasil, mesmo com o desenvolvimento ocorrido nos últimos anos na área farmacológica e nas medidas de saneamento básico e de educação sanitária. As doenças causadas por esses endoparasitas são das mais frequentes em todo o território nacional. Essas enfermidades têm avançado devido à sua facilidade de disseminação, afetando a saúde humana e animal, trazendo um grande impacto à saúde pública e conseguintemente à sociedade e à economia (OIE, 2015).

Segundo o Global Enteric Multicenter Study (GEMS) e o Global Burden of Disease (GBD, 2017), estudos realizados para identificar a etiologia e a carga parasitária em crianças com diarréia associada à mortalidade de crianças menores de cinco anos, tiveram a criptosporidiose como a responsável por 60.000 mortes em 2015 (Kotloff et al., 2013; GBD, 2017). A crescente preocupação com estas doenças se deve à sua rápida e fácil disseminação, através da água contaminada, alimentos contaminados por esterco, qualquer fase de produção de alimentação onde haja águas contaminadas ou utensílios utilizados ou a falta de cuidados por parte dos funcionários (Mawlyet al., 2015; Ryan et al., 2014; Widerström et al., 2014; OIE, 2015).

O Cryptosporidium spp pode ser encontrado em qualquer lugar e tem ampla diversidade de hospedeiros, podendo ter seu desenvolvimento em mamíferos, aves, répteis e anfíbios, nessas espécies o parasita pode causar desde infecções freqüentes a recorrentes subclínicas e clínicas com sinais gastrointestinais (QI et al., 2011; Ran et al., 2014).

A doença pode ser mais leve ou severa, dependendo dos lugares onde o parasita se instala (intestino, lúmen, aparelho respiratório), a condição nutricional, o estado imunológico do indivíduo e a idade do hospedeiro (Chalmers, Davies, 2010). A única forma de contaminação é por meio de sua forma infectante, o oocisto, que é resistente e permanece infectante por longos períodos no ambiente e na água (Thompson et al., 2009). Apesar dos avanços na desinfecção e no monitoramento de qualidade da água, os métodos existentes mostram-se ineficientes na detecção e eliminação desses protozoários, pois esse tipo de doença tem grande velocidade de disseminação, desafiando todos os mecanismos de controle (Meireles, 2010, ReboredoFernandez et al., 2015).

A dificuldade em sua identificação, a natureza mutável do patógeno, a ampla diversidade de hospedeiros, sua forma de transmissão, nos faz reconhecer que são imprescindíveis novas investigações com o objetivo de elucidar qualquer mudança 
em seu padrão e sua real epidemiologia (Baldursson, Karanis, 2011; Mackey et al., 2014). Cada tentativa e esforço na procura pela compreensão dessa doença e de sua emergência do ponto de vista da saúde pública são fundamentais para a prevenção, para o tratamento e para o desenvolvimento de políticas de prevenção e de controle (Mackey et al., 2014; OIE, 2015).

A criptosporidiose é uma doença de extrema prevalência e de grande abrangência com comprovação em mais de 1.000 relatos em seres humanos em 106 países em todos os continentes, com exceção da Antártica (Ryan et al., 2014), porém sua incidência exata em nível mundial não é conhecida (Widerstrom et al., 2014).

Dada a sua importância na saúde pública, sua ocorrência em cinco continentes, afetando tanto humanos e animais, sua alta veiculação pelos alimentos, água e solo que contribuem para o ciclo do parasita, esses fatores favorecem para que a criptosporidiose tenha sido adicionada a World Health Organization's (WHO), "Neglected Diseases Initiative", que inclui doenças que afetam pessoas com poucos recursos e que são negligenciadas pelo estado (Saviolli, Smith, Thompson, 2009).

Supõe-se uma alta variação de sua prevalência devido à subnotificação, à falta de padronização dos métodos diagnósticos, além da falta de acesso a cuidados médicos (Mackey et al., 2014). A criptosporidiose é uma doença de disseminação hídrica, vários relatos e estudos têm mostrado que o oocisto de Cryptosporidium spp é resistente a certa concentração de cloro utilizado no tratamento de água de consumo e de piscina, para inativá-lo são necessárias temperaturas abaixo de $-20^{\circ} \mathrm{C}$ por um período de mais de oito horas (Duhainet al., 2012).

No Brasil, os relatos de casos da doença não têm sido frequentes, tendo o último sido realizado por Castro et al. (2010) em uma creche em São Paulo, estudo que incluiu 224 crianças, das quais 29 (12,9\%) apresentaram oocistos da espécie C. hominis, destacando a prevalência dessa espécie em centros urbanos (Gherasim et al., 2012). Outro estudo efetuado no mesmo ano em creches da cidade de Recife - Pernambuco, com um total de 182 crianças, mostrou que 59 (32,4\%) foram positivas para o Cyptosporidium spp. (Nascimento et al., 2009), em um estudo realizado nas feiras livres com hortaliças no Recife,usando 100 amostras constatou que 12\% delas foram positivas para o parasito (Silva et al., 2005; Rossi et al., 2014).

Conforme Tonani et al. (2013) a forma predominante de tratamento do esgoto no Brasil é o iodo ativado, isso diminui a densidade de oocistos no esgoto (de 28,9 oocistos/L para 1,05 oocistos/L), apesar disso ainda causa problemas a saúde pública, seja no consumo de água ou mesmo na utilização desta para higiene ou recreação (Razzolini et al., 2010). Em 2017, segundo a Agência Brasil de notícias, foram notificadas mais de 263,4 mil internações por doenças decorrentes da falta de saneamento básico e acesso à água no país, gerando um custo de 129 milhões para a sociedade (Agência Brasil, 2020). A Organização Mundial da Saúde estabeleceu a relação entre a saúde pública e o saneamento básico, afirmando que, para cada dólar investido em água e saneamento, é possível economizar 4,3 dólares de custo em saúde (WHO, 2020).

A falta de saneamento básico no Brasil expõe a população a vários riscos à saúde, pesquisas relacionam diversas doenças, ao sistema de água e de esgoto inadequado, causando milhões de mortes todos os anos (Instituto Trata Brasil, 2020). O saneamento básico é uma questão problemática em escala global. Estimou-se, em 2017, que 2,1 bilhões de pessoas (30\% da população mundial) não têm acesso a água potável e 4,4 bilhões (60\% da população mundial) não dispõem de saneamento básico ou condições minimamente satisfatórias (Unicef, 2017).

Dos gestores municipais do Brasil no ano de 2017, 35\% afirmaram saber de epidemias ou endemias de doenças diretamente relacionadas ao saneamento básico. Dentre as doenças mais observadas estavam à diarreia, com 23,1\%, dengue, com 26,9\%, e verminoses, com 17,2\%, todas as doenças citadas são de veiculação hídrica (Agência IBGE Notícias 2020). De acordo com os dados do Sistema de Informação Hospitalar do Ministério da Saúde (SIH/SUS), para o ano de 2013, o estado de Pernambuco registrou 11.872 internações por doença diarreica aguda (DDA), (Rufino et al., 2016).

Conforme o diagnóstico dos Serviços de Água e Esgoto (2015), feito pelo Ministério das Cidades, no Brasil a rede de abastecimento de água totaliza cerca de $83,3 \%$ e sua rede de esgoto chega a $50,3 \%$. O investimento para a melhoria deve 
chegar a 26 bilhões de reais nos próximos 14 anos, com objetivo de alcançar 99\% de abastecimento de água e 92\% de esgotamento sanitário em 2033, conforme o Plano Nacional de Segurança Hídrica e Saneamento Básico (Brasil, 2015).

$\mathrm{Na}$ região nordeste os índices de atendimento com rede de água são de 73\%, enquanto na área urbana chega a $90 \%$ e esgoto no total $25 \%$ e na área urbana de $32 \%$, é a segunda região do país com menores índices de abastecimento de água e rede de esgoto. Esses números mostram a necessidade de maiores investimentos na região em relação à água e esgoto. No ano de 2017 era a segunda região com o pior índice (Instituto Trata Brasil, 2017). O estado de Pernambuco possui 185 municípios, o equivalente a $1,15 \%$ do território brasileiro, com mais de 9,49 milhões de habitantes (4,55\% da população brasileira), o que confere ao estado a décima nona maior área territorial e o sétimo maior contingente populacional dentre os estados do Brasil (IBGE, 2020).

Em Pernambuco, a Portaria da Secretaria Estadual de Saúde n. 390 "trata das doenças, agravos e eventos estaduais à Lista Nacional de Doenças de Notificação Compulsória e dá outras providências”. Em seu anexo II, entre os critérios laboratoriais de notificação/definição de casos, encontra-se a criptosporidiose. Diante do exposto, o objetivo deste estudo é avaliar quais fatores favorecem a ocorrência da criptosporidiose em pacientes atendidos em um hospital universitário da região metropolitana do estado de Pernambuco.

\section{Metodologia}

Trata-se de um estudo retrospectivo, descritivo com abordagem quantitativa, que teve como objeto da pesquisa os prontuários de pacientes atendidos no Hospital Universitário Oswaldo Cruz (HUOC) com solicitação de exames parasitológicos e com diagnóstico de criptosporidiose entre os anos de 2012 à 2020 provenientes do Estado de Pernambuco. A amostra foi obtida do Serviço Médico e Estatístico (SAME) e do Aplicativo de Gestão para Hospitais Universitários (AGHU) do Hospital Universitário Oswaldo Cruz (HUOC).

Para seleção da amostra foram adotados como critério de inclusão os prontuários de pacientes com diagnóstico de criptosporidiose no ano de 2012-2020 do Estado de Pernambuco. Os critérios de exclusão foram: prontuários de pacientes sem confirmação diagnóstica, prontuários ilegíveis, com rasuras e não preenchidos corretamente, pacientes de outros estados. Sendo excluídos (24) prontuários que não atenderam os critérios de inclusão estabelecidos, o que reduziu a amostra a um total de duzentos e setenta e um (247) prontuários, sendo, destes oitenta e nove (89) casos confirmados para a doença. Para um melhor entendimento, e sistematização dos resultados, foi utilizado o quadro vigente do Instituto Brasileiro de Geografia e Estatística (IBGE), entre 1989 - 2017, o qual divide o estado de Pernambuco em cinco mesorregiões: Sertão, Sertão do São Francisco, Agreste, Zona da Mata e Região metropolitana do Recife (IBGE, 2020). 
Figura 1. Mesorregiões do estado de Pernambuco de acordo com Instituto Brasileiro de Geografia e Estatística - IBGE.

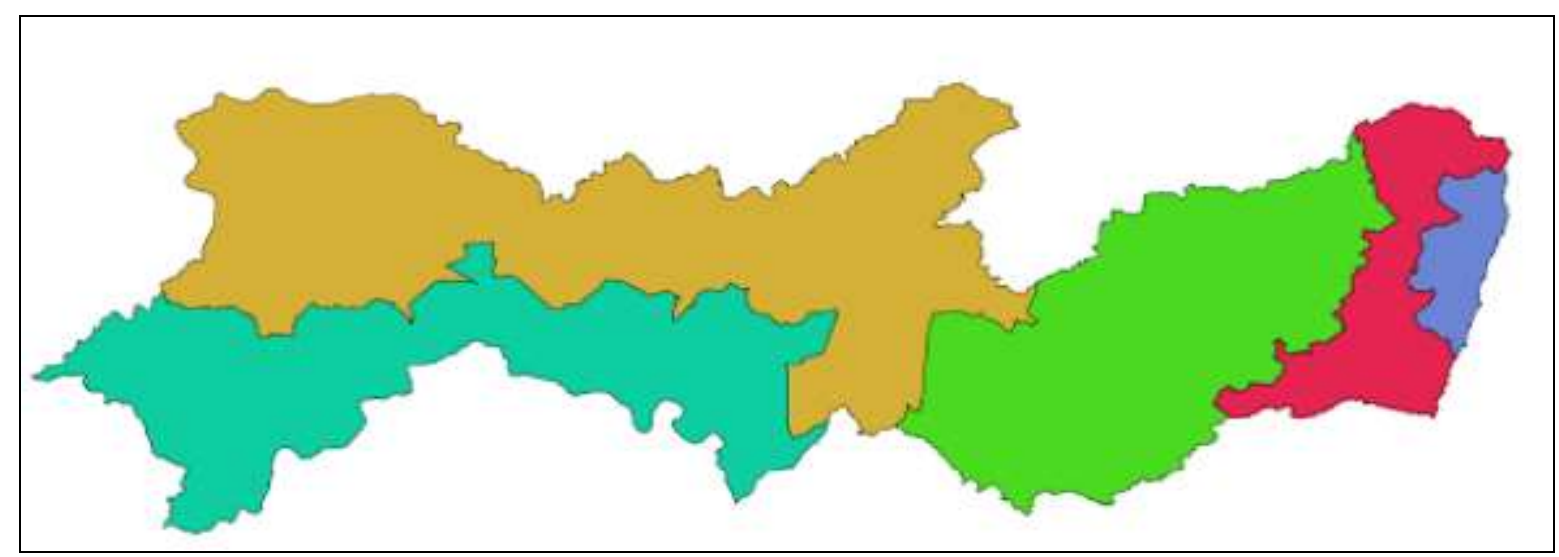

Legenda: - Agreste Pernambucano; $\square$ Zona da Mata Pernambucano; $\square$ Região Metropolitana do Recife; Sertão do São Francisco; $\square$ Sertão Pernambucano. Fonte: IBGE (2020).

Após a coleta, os dados foram organizados e tabulados em planilhas no programa Excel versão Microsoft Office 365 e analisados utilizando-se de medidas descritivas: porcentagem regressão. Para as análises estatísticas foram utilizados os softwares Gretl e Excel, Teste $t$ de Student pareado para as quais foi utilizado, quando aplicável, o nível de significância de 0,05. Foi realizada uma análise descritiva a fim de verificar as características gerais, bem como identificar e apontar as medidas de tendência da amostra. Adicionalmente, foi realizada uma regressão linear com o objetivo de identificar possíveis relações dessa natureza entre as variáveis constantes na equação:

$$
C R Y P=\alpha_{0}+\alpha_{1} N_{-} \text {UPAS }+\alpha_{2} \text { GERES }+\alpha_{2} A N O+\alpha_{4} L N_{-} P O P(1)
$$

A variável CRYP corresponde ao número de casos de criptosporidiose estratificados por Gerência Regional de Saúde (Geres) (variável independente). Como variáveis dependentes foram escolhidos o total de Unidades de Pronto Atendimento (UPAS), de cada Geres (N_UPAS), o número de referência de cada Geres (Gerências regionais de saúde), o ano de referência (ANO) e o logaritmo natural da população de cada Geres (LN_POP).

O estudo foi efetuado de acordo com as Diretrizes e Normas Reguladoras de Pesquisas Envolvendo Seres Humanos, conforme a Resolução 466/12 do Conselho Nacional de Saúde e aprovado pelo comitê de ética em seres humanos pelo parecer n. 3.323.703 de maio de 2019 .

\section{Resultados}

Os diagnósticos de criptosporidiose foram realizados no Laboratório de Parasitologia do Instituto de Ciências Biológicas da Universidade de Pernambuco (ICB/UPE), que atende ao Hospital Universitário Oswaldo Cruz, referência em doenças infectocontagiosas. As amostras que foram encaminhadas através dos ambulatórios e enfermarias do referido hospital, foram analisadas pela metodologia de Ritchie e Kinyoun (Allen, Ridley, 1970; Ritchie, 1948).

A tabela 1 apresenta a distribuição de criptosporidiose nos pacientes do HUOC, observa-se que dos 247 prontuários avaliados 155 são do sexo masculino $(62,8 \%)$ e 92 do feminino (37,2\%), quanto aos prontuários com diagnóstico confirmado de criptosporidiose 89 casos, $58,4 \%$ eram de homens e $41,6 \%$ de mulheres, onde podemos constatar não existe diferença estatística entre os sexos. 
Tabela 1. Distribuição por sexo, idade e ocorrência de Criptosporidiose diagnosticada no HUOC (2012-2020), segundo registros nos prontuários. Recife, PE, Brasil, 2020.

\begin{tabular}{lcccc}
\hline & \multicolumn{3}{c}{ Total } \\
\cline { 2 - 5 } Variável & \multicolumn{2}{c}{ Prontuários } & \multicolumn{2}{c}{ Casos positivos } \\
\hline nomem & n. & $\%$ & 155 & $\%$ \\
\hline Mulher & 155 & $62,8 \%$ & $92,8 \%$ \\
Total & 92 & $37,2 \%$ & 247 & $37,2 \%$ \\
\hline
\end{tabular}

$* t$ de Student $(\mathrm{P}<0,05)$. Fonte: Autores (2020).

Na Tabela 2 verifica-se que os casos de criptosporidiose ocorreram em diferentes idades, mas que não apresentaram diferenças significativas entre os grupos.

Tabela 2. Distribuição dos pacientes atendidos por Criptosporidiose no HUOC nos anos de 2012 a 2020.

\begin{tabular}{lccccc}
\hline Idade & $\begin{array}{c}\text { Total de } \\
\text { Pacientes }\end{array}$ & $\begin{array}{c}\text { Casos } \\
\text { Positivos }\end{array}$ & $\begin{array}{c}\text { Média } \\
\text { Idade }\end{array}$ & $\begin{array}{c}\text { Desvio } \\
\text { Padrão }\end{array}$ & $\begin{array}{c}\text { Casos Positivos } \\
\text { / Total }(\%)\end{array}$ \\
\hline $\begin{array}{l}\text { Crianças } \\
(\mathbf{0 - 1 2} \text { anos })\end{array}$ & 18 & 5 & 7,3 & 3,4 & 5,6 \\
Jovens & 22 & 2 & 22,8 & 5,4 & 2,2 \\
$(\mathbf{1 3 - 2 9}$ anos) & 169 & 67 & & & 75,3 \\
$\begin{array}{l}\text { Adultos } \\
(\mathbf{3 0 - 5 9} \text { anos) }\end{array}$ & & & 44,2 & 8,1 & 16,9 \\
$\begin{array}{l}\text { Idosos } \\
(60+\text { anos })\end{array}$ & 38 & 15 & 71,5 & 11,9 & 100 \\
Total & 247 & 89 & 36,5 & 18,0 & \\
\hline
\end{tabular}

Fonte: Autores (2020).

Na Tabela 3 podemos observar que nos anos compreendidos entre 2012 e 2020 foram diagnosticados no HUOC 89 casos positivos de criptosporidiose, desses quatro (4) casos foram provenientes do Sertão Pernambucano, um (1) do Sertão do São Francisco, três (3) do Agreste, dez (10) da Zona da Mata e setenta e um (71) casos na Região Metropolitana do Recife, essas duas regiões registram os maiores índices de chuvas acumuladas e pluviometria sempre acima do normal. Os maiores valores de precipitação de chuvas ocorreram no litoral, com diminuição gradativa de chuvas à medida que se adentra ao continente, o que pode ser demonstrado com o número reduzido de casos e o pior período de seca da história no sertão nordestino registrado (INMET, 2020). 
Tabela 3. Ocorrência de casos diagnosticados no HUOC em relação à origem dos pacientes por mesorregiões do Estado de Pernambuco nos anos de 2012 a 2020.

\begin{tabular}{|c|c|c|c|c|c|c|c|c|c|c|}
\hline Mesorregião & 2012 & 2013 & 2014 & 2015 & 2016 & 2017 & 2018 & 2019 & 2020 & Total \\
\hline $\begin{array}{l}\text { Sertão } \\
\text { Pernambucano }\end{array}$ & 1 & 0 & 1 & 0 & 0 & 0 & 2 & 0 & 0 & 4 \\
\hline $\begin{array}{l}\text { São Francisco } \\
\text { Pernambucano }\end{array}$ & 0 & 1 & 0 & 0 & 0 & 0 & 0 & 0 & 0 & 1 \\
\hline $\begin{array}{l}\text { Agreste } \\
\text { Pernambucano }\end{array}$ & 1 & 1 & 1 & 0 & 0 & 0 & 0 & 0 & 0 & 3 \\
\hline $\begin{array}{l}\text { Zona da Mata } \\
\text { Pernambucana }\end{array}$ & 1 & 4 & 0 & 1 & 2 & 0 & 2 & 0 & 0 & 10 \\
\hline $\begin{array}{l}\text { Região } \\
\text { Metropolitana }\end{array}$ & 23 & 13 & 4 & 0 & 11 & 3 & 7 & 4 & 6 & 71 \\
\hline Total & 26 & 19 & 6 & 1 & 13 & 3 & 11 & 4 & 6 & 89 \\
\hline
\end{tabular}

Fonte: Autores (2020).

\section{Discussão}

A criptosporidiose apresenta-se agressiva em idosos, diabéticos, crianças e em indivíduos imunocomprometidos. Sendo relacionada aos surtos epidêmicos de diarreias que o coccídeo causa nos indivíduos menores de três anos de idade, adultos não-imunes e idosos (Spósito Filha e Oliveira, 2009).

Dados encontrados neste estudo (Tabelas 1, 2 e 3) divergem do relatado por por Kuiava, Perin e Chielle (2019), que fizeram um levantamento quanto às internações e à mortalidade por diarreia no Brasil no período de 2000 - 2015, mostrando que o número de casos foram expressivamente maiores nos extremos de idade, sendo que as taxas de internação para as idades de 0 - 4 e de 80 anos ou mais foram de 585 e de 362 casos por 100 mil habitantes, respectivamente, enquanto faixas etárias entre os 20 e 49 anos ficaram com valores entre 40 e 50 internações por 100 mil habitantes. Em nosso estudo os maiores números de casos foram na faixa de 30 a 59 anos (75,3\%) seguido pela faixa etária de 60 anos ou mais (16,9\%) dos casos.

Estudos epidemiológicos abrangendo enteroparasitoses são comuns em crianças, porém em adultos e idosos não são frequentes (esse dado traz um alerta à desvalorização das queixas de diarréia por parte dos médicos, e que pode estar associado a um número expressivo de mortes da população adulta e idosa (Laranjeira, Eiriz, Santos, 2014; Santos; Merlini, 2010).

De acordo com o Sistema de Informação Hospitalar do Ministério da Saúde (SIH-SUS) as taxas de hospitalização por diarréia até o ano de 2015 foram de 206,9 a maior dentre todas as regiões brasileiras (Brasil, 2020). Estudos indicam que a diarreia está em primeiro lugar entre as doenças causadas por fatores ambientais e que $94 \%$ da carga das doenças diarreicas pode ser atribuída às condições ambientais (Prüss-Üstün, Corvalán, 2007). Em 2017 o Fundo das Nações Unidas para a infância (Unicef) revelou que 1,7 milhões de óbitos anuais de crianças no mundo estão relacionados ao meio ambiente e 361 mil crianças menores de cinco anos morrem em razão de diarreia, como resultado do baixo acesso à água tratada, ao saneamento e a condições inadequadas de higiene (UNICEF, 2017). Os dados obtidos em nosso estudo (tabela 2) revelam que a população entre 0-12 anos (crianças) teve uma taxa baixa de infecção $(5,6 \%)$ dos casos, acima apenas da faixa de 13 a 29 anos com $2,2 \%$.

Conforme Asfora, Lima e Lacerda (2017) o estado de Pernambuco possui 89\% de sua área com clima semi-árido e os outros $11 \%$ com clima úmido e subúmido, de acordo com a classificação climática de Thornthwaite. O clima semi-árido, 
presente nas mesorregiões do Sertão e Agreste, caracteriza-se pelos baixos índices pluviométricos, com longos períodos secos e chuvas escassas, concentradas em poucos meses do ano e altas temperaturas. A pluviosidade média mantém-se entre 500 e 800 milímetros (mm) anuais. O número reduzido de casos de criptosporidiose nas regiões do Agreste, Sertão de Pernambuco e do São Francisco entre os anos de 2012 a 2020 (Tabela 3) com o total de oito (8) casos pode estar relacionado aos baixos índices de chuvas nestas mesorregiões. De acordo com os dados da Dados da Agência Pernambucana de Águas e Clima (APAC) que mostram que nos anos de 2012 a 2020 ocorreram redução dos níveis das chuvas em relação ao total anual médio em todo o Estado, sendo observados desvios em relação à média de aproximadamente 40 a $80 \%$ no Agreste, Sertão pernambucano e do São Francisco (Boletim APAC, 2012-2020; Asfora, Lima, Lacerda, 2017).

No ano de 2019 a APAC registrou os maiores valores de precipitação de chuvas no litoral, e em 2020 a RMR apresentou um acumulado de chuva na ordem de $1403 \mathrm{~mm}$, bem acima das de outras mesorregiões do Estado (Boletim APAC, 2020). De acordo com APAC dentre todas as mesorregiões do estado de Pernambuco as da Zona da Mata e Região Metropolitana do Recife são as que apresentam maior quantidade de chuvas para o período estudado (Boletim APAC, 20122020). Esse dado mostra a relação entre as chuvas ocorridas nessas duas regiões com o número (81) de casos de criptosporidiose registrados no mesmo período (tabela3).

De acordo com Asfora, Lima, Lacerda (2017) a região metropolitana do Recife e a Zona da Mata têm clima tropical úmido, que apresenta baixas amplitudes térmicas, alta umidade relativa do ar e precipitações médias anuais entre 1.500 e 2.500 $\mathrm{mm}$, essas regiões possuem período chuvoso extenso, com pelo menos seis meses de duração. Conforme Souza et al. (2018) $a$ temperatura e a umidade, trazem efeitos sobre o ciclo biológico de agentes infecciosos, parasitários e seus vetores, dando condições adequadas para os processos de infecção (Sousa, Amancio, Hacon, \& Barcellos, 2018).Nos anos de 2013 e 2016 ocorreram períodos de chuvas intensos nessas regiões, o que coincidiu com o aumento do número de casos de criptosporidiose, sendo registrados 35 casos positivos. Assim, percebe-se uma relação direta entre chuvas e criptosporidiose, corroborado com Guzman Herrador et al. (2015), que explicam existir uma associação entre o aumento na precipitação de chuvas com o aumento nos casos de doenças de veiculação hídrica.

Em 2005 o Ministério da saúde editou o plano Nacional de vigilância e controle para a definição de estratégias entre os estados e municípios, onde cada estado segue as diretrizes desse plano através das secretárias de saúde (Soares, 2018). Em Pernambuco existem doze (12) Geres - unidades administrativas da Secretaria Estadual de Saúde (SES) para apoiar todos os cento e oitenta e quatro (184) municípios do estado mais o distrito de Fernando de Noronha para atuar em partes do Estado, em especial na atenção básica, na rede hospitalar, nas ações municipais, no combate à mortalidade infantil e às diversas endemias. Esse modelo implantado ajuda na avaliação de cada região, suas particularidades e, a partir disso, a decidir sobre ações e campanhas direcionadas aos municípios pernambucanos (SES, 2020).

A Política Nacional de Urgência (PNAU), em parceria com várias entidades de saúde e o governo federal, criou as Unidades de Pronto Atendimento (UPAs), como ponto de acesso ao sistema de saúde, com urgência pré-hospitalar, unidade intermediárias entre a atenção básica e as emergências hospitalares, para desafogar as urgências e emergência e reduzir os indicadores de morbi-mortalidade (O'dwyer, 2010; Brasil, 2011).

Ao se analisarem o número de casos de criptosporidiose estratificados por número de Geres existentes no Estado de Pernambuco constatou-se que o número de UPAs tem influência sobre o total de casos de criptosporidiose. A RMR pertencente a Geres 1, composta de 20 municípios localizados no litoral e na zona da mata do estado, possuindo o maior número de Unidades de Pronto Atendimento (UPAS), sendo a capital Recife o segundo pólo médico do país e o que apresentou maior número de casos de criptosporidiose durante o período de 2012 a 2020 (SES, 2020). Com base na regressão (1), não se 
pode rejeitar a hipótese de que cada UPA a mais na Geres indica uma chance de cerca de 70\% de chance de diagnosticar um caso de criptosporidiose.

Por outro lado, a falta de conhecimento pode levar a falha no processo de diagnóstico, e, nesse sentido, Wiebbelling et al. (2002) explicam que muitos médicos acreditam que a solicitação de um exame parasitológico de rotina é capaz de identificar todos os parasitas. De acordo com Quadros e Miguel (2018) a técnica de exames para a detecção dos oocistos do Cryptosporidium spp. é específica e não faz parte da rotina, tornando o diagnóstico baixo e subestimado na população. Gerando uma subnotificação da ocorrência do caso, prejudicando a atualização dos dados epidemiológicos e o não ajuste dos programas de saúde pública, principalmente se levarmos em conta que o parasito é considerado um problema não só nos países subdesenvolvidos, mas nos desenvolvidos também (Domjahn et al., 2013). Para o diagnóstico da criptosporidiose são necessários dois métodos: o método de Ritchie, onde é feita a concentração dos oocistos, e o método de Kinyoun, de coloração, porém esses exames não fazem parte dos exames de rotina das unidades hospitalares ou dos laboratórios, pois requer um exame diferenciado (Ross et al., 2009).

Assim, a doença pode estar sendo subestimada, especialmente em crianças, adultos e idosos. Muitos médicos acreditam que a solicitação de um exame parasitológico de rotina identifica todos os parasitas. Um comunicado da Organização Mundial de Saúde (ECODEBATE, 2020) sobre mudança climática, informa, afirma, e declara que doenças infecciosas e ameaças de epidemia, desigualdades socioeconômicas e conflitos são alguns dos 13 desafios urgentes que a OMS afirma colocar em risco a saúde global, mas abordá-los está "ao alcance" se as medidas forem tomadas agora. Uma das medidas citadas é o cuidado com a água, com o saneamento e com a higiene.

\section{Conclusões}

Este trabalho observou que há relação entre chuvas e ocorrência da criptosporidiose. Isso é notado na relação entre a mesorregião da Região Metropolitana do Recife (RMR), onde o registro de número de casos e a ocorrência da maior quantidade de chuvas estão relacionados. Nas mesorregiões do sertão, do sertão do São Francisco, do agreste e da zona da mata, onde ocorreram secas ou chuvas esporádicas, o número foi bem menor. Deve-se atentar ainda para o fato de que o período de análise da pesquisa coincidiu com o maior período de seca registrado, de 2012 a 2018, para essas mesorregiões.

O Nordeste é a região do país com o segundo menor índice de saneamento básico e internação por diarreia, só perdendo para a região norte. Pode-se concluir também que quanto maior o número de unidades de pronto atendimento, maior o número de registro de casos e que há uma falta de comunicação entre os registros de casos e Centro de Informações Estratégicas de Vigilância em Saúde de Pernambuco - Cievs/PE, que pode indicar a existência de um número ainda maior de casos, porém sem a correta identificação ou notificação, levando assim a uma limitação do estudo.

Em continuidade a este trabalho, sugere-se a realização de estudos de observação contínua das internações por diarreia a fim de se detectarem alterações nos padrões sazonais de predominância dos casos da doença, notadamente no estado de Pernambuco. Os resultados alcançados podem auxiliar na caracterização e no planejamento de ações de prevenção das diarréias nas regiões abordadas, além de auxiliar na orientação das unidades de saúde para uma melhor identificação e notificação da doença.

O estudo mostra a importância de uma melhoria nos sistemas de saúde pública na prevenção da doença e no preparo para o atendimento e tratamento dos casos, principalmente em um período em que se observam cada vez mais eventos climáticos extremos, além de os prognósticos ligados às mudanças do clima apontar para grandes impactos sobre a saúde das populações. 
Research, Society and Development, v. 10, n. 12, e571101220459, 2021 (CC BY 4.0) | ISSN 2525-3409 | DOI: http://dx.doi.org/10.33448/rsd-v10i12.20459

\section{Referências}

Agência Brasil (2018). Doenças ligadas à falta de saneamento geram custo de r\$100 mil ao SUS: levar tratamento de esgoto para população é desafio dos governantes. Brasília. <http://agenciabrasil.ebc.com.br/saude/noticia/2018-09/doencas-ligadas-falta-de-saneamento-geram-custo-de-r-100-mi-ao-sus〉.

Allen, A. V., \& Ridley, D. S. (1970). Further observations on the formol-ether concentration technique for faecal parasites. Journal of clinical pathology, 23(6):545.

Asfora, M. C., Lima, M., \&Lacerda, M. R. D. S. (2017). Diagnóstico da seca 2011-2016 em Pernambuco: impactos e políticas de mitigação. ParceriasEstratégicas, 22(44):247-273.

Baldursson, S., \&Karanis, P. (2011). Waterborne transmission of protozoan parasites: review of worldwide outbreaks-an update 2004-2010. Waterresearch, 45(20):6603-6614.

Brasil (2018). Ministério da Saúde - MS.DataSUS. Brasília: Departamento de Informática do Sistema Único de Saúde. Brasília: Ministério da Saúde. <www.datasus.saude.gov.br>.

Brasil Ministério da Saúde (2011). Portaria n 2.648, de 7 de novembro de 2011. Redefine as diretrizes para implantação do Componente Unidade de Prontoatendimento (UPA 24h) e do conjunto de serviços de urgência 24 (vinte e quatro) horas da Rede de Atenção às Urgências, em conformidade com a Política Nacional de Atenção às Urgências. Diário Oficial da União, Brasília, DF, 8 nov. 2011. p. 48.

Brasil Ministério do Desenvolvimento Regional - MDR (2018). Secretária Nacional de Saneamento - SNS. Sistema Nacional de Informações sobre Saneamento - SNIS.24 Diagnóstico dos serviços de água e esgotos - 2018. Brasília, DF: MDR/SNS, 2019. <http://www.snis.gov.br> .

Castro, J. M., Cipollari, P. M., Seliger, S. M. P. A., Conde, R. L. A., Soares, V. C., \& Ribeiro, M. A. C. (2010). surto de criptosporidiose em creche do distrito administrativo de Vila Guilherme, zona norte de São Paulo/SP. Revista Saúde-UNG-Ser, 4(1 Esp):70.

Centro de Informações Estratégicas de Vigilância em Saúde de Pernambuco - CIEVS/PE, 2020. <http://cievspe.blogspot.com/search?q=cryptosporidium>.

Chalmers, R. M., \& Davies, A. P. (2010). Minireview: clinicalcryptosporidiosis. Experimental parasitology, 124(1):138-146.

Diarrhoel Diseases Colllaborators - GBD (2017). Estimates of global, regional, and national morbidity, and aetiologies of diarrhoeal diseases: a systematic analysis for the global Burden of Disease Study 2015, Lancet Infect Dis,17(9): 909-948, 2017.

Domjahn, B. T., Hlavsa, M. C., Anderson, B., Schulkin, J., Leon, J., \& Jones, J. L. (2014). A Survey of US Obstetrician-Gynecologists' Clinical and Epidemiological Knowledge of Cryptosporidiosis in Pregnancy. Zoonoses and public health, 61(5):356-363.

Ecodebate (2020). Site de informações, artigos e notícias socioambientais. OMS alerta para 13 ameaças emergentes à saúde, incluindo possíveis pandemias. $<$ https://www.ecodebate.com.br/2020/01/23/oms-alerta-para-13-ameacas-emergentes-a-saude-incluindo-possiveis-pandemias/>.

Efstratiou, A; Ongerth, J. E; Karanis, P. (2017). Waterborne transmission of protozoan parasites: Review of worldwide outbreaks - An update 2011-2016. WaterResearch, 1(114:(14-22).

Fundo das Nações Unidas para Infância - UNICEF (2019). 1 em cada 3 pessoas no mundo não tem acesso a água potável, dizem o UNICEF e a OMS, 2019. <https://www.unicef.org/brazil/comunicados-de-imprensa/1-em-cada-3-pessoas-no-mundo-nao-tem-acesso-agua-potavel-dizem-unicef-oms>.

Gherasim, A., Lebbad, M., Insulander, M., Decraene, V., Kling, A., Hjertqvist, M., \& Wallensten, A. (2012). Two geographically separated food-borne outbreaks in Sweden linked by an unusual Cryptosporidium parvum subtype, October 2010. Eurosurveillance,17(46), 20318.

Gonçalves, R. F., Sousa, I. M. C. D., Tanaka, O. Y., Santos, C. R. D., Brito-Silva, K., Santos, L. X., \& Bezerra, A. F. B. (2016). Programa Mais Médicos no Nordeste: avaliação das internações por condições sensíveis à Atenção Primária à Saúde. Ciência \& Saúde Coletiva, 21, $2815-2824$.

Graczyk, T. K., Majewska, A. C., \&Schwab, K. J. (2008). The role of birds in dissemination of human waterborne enteropathogens. Trends in parasitology, 24(2), 55-59.

Herrador, B. R. G., De Blasio, B. F., MacDonald, E., Nichols, G., Sudre, B., Vold, L., \&Nygård, K. (2015). Analytical studies assessing the association between extreme precipitation or temperature and drinking water-related waterborne infections: a review. Environmental Health, 14(1), 1-12.

Instituto Brasileiro de Geografia e Estatística - IBGE. Agência IBGE Notícias. Estatísticas Sociais, 2018. http://agenciadenoticias.ibge.gov.br/agencia-sala-deimprensa/2013-agencia-de-noticias/releases/22611-munic-mais-da-metade-dos-municipios-brasileiros-nao-tinha-plano-de-saneamento-basico-em-2017.

Instituto Brasileiro de Geografia e Estatística - IBGE. Divisão regional do Brasil em mesorregiões e microrregiões geográficas (1990). Rio de Janeiro, RJ: IBGE, p. 52-55. <https://biblioteca.ibge.gov.br/visualizacao/monografias/GEBIS\%20-\%20RJ/DRB/Divisao\%20regional_v01.pdf>.

Instituto Nacional de Meteorologia - INMET (2020). <http://www.inmet.gov.br/portal/index.php?r=home/page\&page=sobre_inmet> .

Instituto Trata Brasil (2018). Manual do saneamento básico: entendendo o saneamento básico ambiental no Brasil e sua importância socioeconômica, 2018. <http://www.tratabrasil.org.br/datafiles/uploads/estudos/pesquisa16/manual-imprensa.pdf>.

Kotloff, K. L., Nataro, J. P., Blackwelder, W. C., Nasrin, D., Farag, T. H., Panchalingam, S., \& Levine, M. M. (2013). Burden and aetiology of diarrhoeal disease in infants and young children in developing countries (the Global Enteric Multicenter Study, GEMS): a prospective, case-control study. The Lancet, 382(9888): 209-222.

Kuiava, V. A., Perin, A. T., \&Chielle, E. O. (2019). Hospitalização e taxas de mortalidade por diarreia no Brasil: 2000-2015. Ciência \& Saúde, 12(2):e30022e30022. 
Research, Society and Development, v. 10, n. 12, e571101220459, 2021 (CC BY 4.0) | ISSN 2525-3409 | DOI: http://dx.doi.org/10.33448/rsd-v10i12.20459

Laranjeira, C., Eiriz, R., \& Santos, P. (2014). Vacinação contra o rotavírus nos cuidados de saúde primários. Revista Portuguesa de Medicina Geral e Familiar, 30(2):78-82.

Mackey, T. K., Liang, B. A., Cuomo, R., Hafen, R., Brouwer, K. C., \& Lee, D. E. (2014). Emerging and reemerging neglected tropical diseases: a review of key characteristics, risk factors, and the policy and innovation environment. Clinical microbiology reviews, 27(4):949-979.

Meireles, M. V. (2010). Cryptosporidium infection in Brazil: implications for veterinary medicine and public health. Revista Brasileira de Parasitologia Veterinária, 19(4):197-204.

Messias, M. G., \& Neves, R. D. F. (2009). A influência de fatores comportamentais e ambientais domésticos nas quedas em idosos. Revista brasileira de geriatria e gerontologia, 12(2):275-282.

Nascimento, W. R. C. D., Cavalcanti, I. M. F., Irmão, J. I., \& Rocha, F. J. S. (2009). Presença de Cryptosporidiumspp em crianças com diarréia aguda em uma creche pública de Recife, Estado de Pernambuco. Revista da Sociedade Brasileira de Medicina Tropical, 42(2):175-178

O'Dwyer, G. (2010). A gestão da atenção às urgências e o protagonismo federal. Ciência \& saúde coletiva, 15(5):2395-2404

Organização Mundial da Saúde - OMS (2016). Expectativa de vida sobe 5 anos de 2000 a 2015 no mundo, mas desigualdades persistem. <https://nacoesunidas.org/oms-expectativa-de-vida-sobe-5-anos-de-2000-a-2015-no-mundo-mas-desigualdades-persistem/>

Organização Mundial da Saúde - OMS (2014). Para cada dólar investido em água e saneamento, economiza-se 4,3 dólares em saúde global. Geneva: WHO. <https://nacoesunidas.org/oms-para-cada-dolar-investido-em-agua-e-saneamento-economiza-se-43-dolares-em-saude-global/>.

Pernambuco (Estado) (2020). Agência Pernambucana de Água e Clima - APAC. Boletim 2012-2020. <http://www.apac.pe.gov.br/>

Pernambuco (Estado) (2016). Portaria $\mathrm{n}^{\circ}$. 390, de 14 de setembro de 2016. Acrescenta doenças, agravos e eventos estaduais à lista nacional de doenças de notificação compulsória e dá outras providências. Diário Oficial do Estado, Recife: Secretaria Estadual de Saúde, 14 set. 2016. <https://www.cievspe.com/deteccao>.

Pernambuco (Estado) (2020). Secretaria Estadual de Saúde. Institucional Recife, 2020. Disponível em: <http://portal.saude.pe.gov.br/secretaria>. Acesso em: 2 fev. 2020

Prüss-Üstün, A., \&Corvalán, C. (2007). How much disease burden can be prevented by environmental interventions? Epidemiology, 18(1):167-178.

Quadros, R. M. D., \& Miguel, R. D. L. (2018). Cryptosporidium spp. em idosos residentes em instituições de longa permanência no sul do Brasil. Clin. Biomed. Res, 38(3):223-228.

Qi, M., Wang, R., Ning, C., Li, X., Zhang, L., Jian, F., Sun, Y.\& Xiao, L. (2011). Cryptosporidium spp. in pet birds: genetic diversity and potential public health significance. Experimental Parasitology, 128(4):336-340.

Razzolini, M. T. P., da Silva Santos, T. F., \& Bastos, V. K. (2010). Detection of Giardia and Cryptosporidium cysts/oocysts in watersheds and drinking water sources in Brazil urban areas. Journal of water and health, 8(2):399-404.

Reboredo-Fernandez, A., Ares-Mazas, E., Caccio, S. M., \& Gomez-Couso, H. (2015). Occurrence of Giardia and Cryptosporidium in wild birds in Galicia (Northwest Spain). Parasitology, 142(7):917-925.

Ritchie, L. S. (1948). An ether sedimentation technique for routine stool examinations. Bulletin of the United States Army medical department, 8(4):326.

Ross, D. S., Rasmussen, S. A., Cannon, M. J., Anderson, B., Kilker, K., Tumpey, A., \& Jones, J. L. (2009). Obstetrician/gynecologists' knowledge, attitudes, and practices regarding prevention of infections in pregnancy. Journal of Women's Health, 18(8): 1187-1193.

Rossi, G. A. M., Hoppe, E. G. L., Martins, A. M. C. V., \&Prata, L. F. (2014). Zoonoses parasitárias veiculadas por alimentos de origem animal: revisão sobre a situação no Brasil. Arquivos do Instituto Biológico, 81(3):290-298.

Rufino, R., Gracie, R., Sena, A., Freitas, C. M. D., \& Barcellos, C. (2016). Surtos de diarreia na região Nordeste do Brasil em 2013, segundo a mídia e sistemas de informação de saúde-Vigilância de situações climáticas de risco e emergências em saúde. Ciência \& Saúde Coletiva, 21(3): 777-788.

Ryan, U. N. A., Fayer, R., \&Xiao, L. (2014). Cryptosporidium species in humans and animals: current understanding and research needs.Parasitology, 141(13):1667-1685.

Santos, S. A. D., \&Merlini, L. S. (2010). Prevalência de enteroparasitoses na população do município de Maria Helena, Paraná. Ciência\&SaúdeColetiva, 15(3):899-905

Savioli, L., Smith, H., \& Thompson, A. (2006). Giardia and Cryptosporidium join the 'neglected diseases initiative'. Trends in parasitology, 22(5):203-208.

Silva, C. G. M. D., Andrade, S. A. C., \& Stamford, T. L. M. (2005). Ocorrência de Cryptosporidium spp. e outros parasitas em hortaliças consumidas in natura, no Recife. Ciência \& Saúde Coletiva, 10(suppl):63-69.

Solano, J. P. C., Scazufca, M., \& Menezes, P. R. (2011). Frequência de sintomas no último ano de vida de idosos de baixa renda em São Paulo: estudo transversal com cuidadores informais. Revista Brasileira de Epidemiologia, 14(1):75-85.

Sousa, T. C. M. D., Amancio, F., Hacon, S. D. S., \& Barcellos, C. (2018). Doenças sensíveis ao clima no Brasil e no mundo: revisão sistemática. Revista Panamericana de Salud Pública, 42:e85. 
Research, Society and Development, v. 10, n. 12, e571101220459, 2021 (CC BY 4.0) | ISSN 2525-3409 | DOI: http://dx.doi.org/10.33448/rsd-v10i12.20459

Spósito-Filha, E., Oliveira, S. M (2009). Divulgação técnica Criptosporidiose. Biológico, 71(1):17-19.

Tonani, K. A. A., Padula, J. A., Julião, F. C., Fregonesi, B. M., Alves, R. I. S., Sampaio, C. F., \& Segura-Muñoz, S. I. (2013). Persistence of giardia, cryptosporidium, rotavirus, and adenovirus in treated sewage in São Paulo state, Brazil. The Journal of parasitology, 99(6):1144-1147.

Widerström, M., Schönning, C., Lilja, M., Lebbad, M., Ljung, T., Allestam, G., \& Lindh, J. (2014). Large outbreak of Cryptosporidium hominis infection transmitted through the public water supply, Sweden. Emerging infectious diseases, 20(4):581.

Wiebbelling, A. M., Mezzari, A., Schenato, L. K., Richter, V. T., \& Bohme, E. S. (2002). Avaliação do conhecimento sobre criptosporidiose em uma amostra de médicos de Porto Alegre, Rio Grande do Sul. Jornal Brasileiro de Patologia e Medicina Laboratorial, 38(2):119-123.

World Organisation for Animal Health - OIE.(2015). Emerging and re-emerging zoonoses <https://www.oie.int/en/for-themedia/editorials/detail/article/emerging-and-re-emerging-zoonoses/>. 